\title{
Venous thromboembolism and pancreatic cancer
}

Abbreviations: DVT, deep vein thrombosis; ECOG, Eastern Cooperative Oncology Group; IVC, inferior vena cava; IVT, intra-abdominal venous thromboembolism; PTE, pulmonary thromboembolism; SMV, superior mesenteric vein; VTE, venous thromboembolism

\section{Introduction}

Pancreatic cancer (PC), one of the most lethal malignancies, is known to be frequently associated with venous thromboembolism (VTE). Studies reported that VTE incidences are different according to cancer type, and they have a common result that PC is one of the malignancies, which is most highly associated with VTE incidence..$^{1,2}$ In the most commonly used predictive model for chemotherapyassociated thrombosis, PC was categorized in "very high risk" group with gastric cancer. ${ }^{3}$ Furthermore, even asymptomatic incidental VTE was also associated with mortality in PC. ${ }^{4}$ These findings suggest that prophylactic anticoagulation should be an important part of treatment for patients with PC.

\section{Methods}

We retrospectively reviewed all patients with pancreatic cancer treated in the Department of Medical Oncology at the Hassan II University Hospital. A total of 120 patients were detected between January 2013 to Juin 2017. This study were adressed to analyze the prevalence of VTE and to investigate risk factors associated with the development of VTE after PC diagnosis.

\section{Results}

Among 120 pancreatic adenocarcinoma, 18 (15\%) patients had a venous thromboembolic disease.

The median age of overall patients was 59.2 with range between 54 and 77. ECOG performance status was 3 and 4 in 30\% cases. More than half of patients $(70 \%)$ had metastatic pancreatic adenocarcinoma and nearly half of them (27.1\%) had multiple metastatic lesions. A total of $23(18.7 \%)$ patients underwent major abdominal surgery with the aim of curative treatment and $72(60 \%)$ received chemotherapy with adjuvant or palliative aims.

Venous thromboembolisms were diagnosed with imaging modalities including Doppler ultrasonography, or computed tomography (CT), depending on the anatomical sites. The asymptomatic and incidental VTE were detected during the assessment scan. 5 (27.7\%) patients had pulmonary thromboembolism (PTE) and $38.8 \%$ of patients in deep vein thrombosis (DVT). The rest VTE events (33.3\%) occurred in the intra-abdominal vessels - portal vein, superior mesenteric vein (SMV), inferior vena cava (IVC), splenic vein, and others.

The diagnosis of DVT was synchronous with the progression of the disease in $16.6 \%$ of cases. 7 patients had VTE and PC simultaneously in the first imaging study. Two of them had previous CT imaging, and both had VTE before PC diagnosis. The potential risk factors associated with developing the VTE are: ischemic heart
Volume 10 Issue $3-2019$

\section{K Messoudi, N Acharfi, K Oualla, S Berrad, I El Ouadki, L Amaadour, Z Benbrahim, S Arifi, $\mathrm{N}$ Mellas}

Medical Oncology Department, Hassan II University Hospital, Faculty of Medicine and Pharmacy of Fez, Morocco

\author{
Correspondence: K Messoudi, Medical Oncology Department, \\ Hassan II University Hospital, Faculty of Medicine and Pharmacy \\ of Fez, Morocco, Tel 00212653744778 , \\ Email kawthar.messoudi@gmail.com
}

Received: June 10,2019| Published: June 20, 2019

disease was presented in $10 \%$ cases. Advanced cancer stage at the time of diagnosis was the strongest risk factor (incidence of VTE was higher in metastatic cancer $55.5 \%$ compared a resectable PC $11.1 \%)$. Treatment was started on the same day of diagnosis and was based on low-molecular-weight heparins (LMWHs) + /- vitamin $\mathrm{K}$ antagonist. Relay to antivitamin $\mathrm{K}$ was established in $33 \%$ of cases. Thromboembolic disease was controlled in $16 \%$ of cases; $70 \%$ have been lost.

\section{Conclusion}

Cancer is a major risk factor for thromboembolic disease, especially for patients with poor general health and advanced cancer. Advanced metastatic stage was the strongest predictor of VTE. Scores carrying predictive value will support the indication of prophylactic anticoagulation for patients at low bleeding risk and high risk for venous thromboembolism. Recent and ongoing clinical trials have focused on VTE prophylaxis with low molecular weight heparins (LMWHs) in high-risk cancer outpatients, particularly those with pancreatic cancer

\section{Competing interest}

The authors declare that they have no competing interests.

\section{References}

1. Marks MA, Engels EA. Venous thromboembolism and cancer risk among elderly adults in the United States. Cancer Epidemiol Biomarkers Prev. 2014;23(5):774-783.

2. Horsted F, West J, Grainge MJ. Risk of venous thromboembolism in patients with cancer: a systematic review and meta-analysis. PLoS Med. 2012;9(7):e1001275.

3. Menapace LA, Peterson DR, Berry A, et al. Symptomatic and incidental thromboembolism are both associated with mortality in pancreatic cancer. Thromb Haemost. 2011;106(2):371-378.

4. Khorana AA, Kuderer NM, Culakova E, et al. Development and validation of a predictive model for chemotherapy-associated thrombosis. Blood. 2008;111(10):4902-4907. 